\title{
Bioequivalence Assessment of Metformin Tablet Formulations of Bangladesh
}

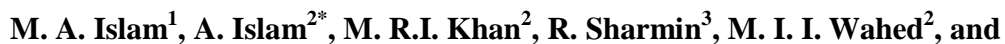 \\ A. S. M. Anisuzzaman ${ }^{2}$ \\ ${ }^{1}$ Square Pharmaceuticals Ltd, Salgaria, Pabna, Bangladesh \\ ${ }^{2}$ Department of Pharmacy, University of Rajshahi, Rajshahi-6205, Bangladesh \\ ${ }^{3}$ Department of Pharmacy, Jessore University of Science and Technology (JSTU), Jessore-7408, \\ Bangladesh
}

Received 25 June 2014, accepted in revised form 20 August 2014

\begin{abstract}
Six marketed oral antidiabetic metformin tablets in Bangladesh have been studied for their drug content, release profile and glucose lowering capacities. This sort of study is a good indicator for in vivo evaluation of the quality of an oral antidiabetic preparation. Marketed preparations of metformin- $\mathrm{HCl}$ from different manufacturers were randomly chosen for this study. The drug content was within the United State Pharmacopoeia (USP) specified limit $(95-105 \%)$ in all cases. The blood glucose levels were investigated in streptozotocin-induced diabetic rats (SIDRs) after 5 hours of single dose $(110 \mathrm{mg} / \mathrm{kg}$ body weight) treatment of the products; significantly $(p<0.05)$ reduced blood glucose level by $58.1,53.2,50.8,77.0,72.9$ and $49.1 \%$, respectively; which were consistent with antihyperglycemic effects of standard metformin- $\mathrm{HCl}(71.3 \%)$. All the products were found to be qualified in lowering blood glucose level. It may be inferred that the metformin- $\mathrm{HCl}$ tablets of Bangladeshi manufacturers complies with the standard specifications for drug contents, dissolution and antihyperglycemic properties.
\end{abstract}

Key words: Metformin; oral hypoglycemic agent; Streptozotocin-induced diabetic rats.

๑) 2014 JSR Publications. ISSN: 2070-0237 (Print); 2070-0245 (Online). All rights reserved.

doi: http://dx.doi.org/10.3329/jsr.v6i3.19290 J. Sci. Res. 6 (3), 581-588 (2014)

\section{Introduction}

Diabetes mellitus is a metabolic disorder characterized by hyperglycemia, abnormal lipid and protein metabolism along with specific long-term complications affecting the retina, kidney and nervous system [1]. Hyperglycemia is an important risk factor in the development and progression of the complication of diabetes mellitus [2]. The pathogenesis of diabetes mellitus is managed by insulin and oral administration of hypoglycemic drugs such as sulfonylureas and biguanides [3].

\footnotetext{
*Corresponding author: paislam@yahoo.com
} 
Biguanides are by-products of guanidine. The discovery of the widely used biguanide antidiabetic drug, metformin ( $\mathrm{N}, \mathrm{N}$-dimethylguanidine) came from the traditional approach through the use of Galega officinalis [4]. Now-a-days although numerous natural remedies are being investigated [5-7] to alleviate the diabetic complications, still its regular control largely depends on chemical approach. Several biguanide derivatives were demonstrated having its utility, therapeutic efficacy, safety, potentiality in cardiovascular benefits, as well as its glucose-lowering effects in the treatment of the type II diabetes by UK Prospective Diabetes Studies (UKPDS) [8]. Now metformin is regarded as a first line treatment of type II diabetes mellitus in national and international guidelines. Metformin given alone or in combination [9] with sulfonylurea improves glycemic control and lipid concentration in patients who respond poorly to diet or to sulfonylureas alone [10].

Among several antidiabetic drugs; metformin is an ideal antihyperglycemic but not hypoglycemic drug [11] because it does not affect insulin release from the pancreas and does not cause hypoglycemia, even in large doses. Metformin reduces glucose levels primarily by decreasing hepatic glucose production and by increasing insulin action in muscle and fat [12]. The mechanism by which metformin reduces hepatic glucose production is controversial but preponderance of data indicates an effect on reducing gluconeogenesis [13].

Pharmaceutical sector in Bangladesh is a second promising sector in recent era [14]. Due to modern technical advances (compliance with several international standards), Bangladesh is now exporting medicines including anti-cancer, anti-diabetic, vaccines for viral diseases, and hormones to US, Europe and Asian countries (around 70 countries) after meeting its local demands [15]. For exporting the products, they must comply several specifications setted by the local supervisory authority and internationals standard organizations (ISO), world health organizations (WHO) and so on. Our current study is a part of series bioequivalence assessment of several kinds of medicines, checking their stability, dissolution characteristics and therapeutic efficacy which are the prerequisite for being a quality medicine in local as well as international pharma market. Metformin is manufactured by several pharmaceutical companies of Bangladesh in different brand names [15], it is extremely important to know the compatibility of the drug and its excipients in formulation which may impair the efficacy of the drugs [16]. Their analytical as well as pharmacological assay is essential for identifying them as quality product. This type of study [17] is a good marker for in vivo evaluation of a quality product. In the present work we use simple techniques for measuring the content of active drug, dissolution characteristics and pharmacological efficacy of these formulations.

\section{Materials and Methods}

\subsection{Materials}

Six metformin- $\mathrm{HCl}$ tablets of different Bangladeshi manufacturers were purchased from local market of Rajshahi, Bangladesh. They were randomly marked as F1 to F6 and 
stored. The labels of all the products were claimed to contain $500 \mathrm{mg}$ of active ingredient per tablet. Metformin-HCl was a generous gift from Square Pharmaceuticals Ltd., Pabna, Bangladesh. Streptozotocin was purchased from Sisco Research Laboratories Pvt. Ltd, Mumbai, India. All other chemicals were of reagent grade.

\subsection{Instruments}

Type-II six-vessel Dissolution Tester (USP). UV spectrophotometer (Shimadzu, UV-1200, UV-VIS spectrophotometer). BioLand G-423 Test Meter (BioLand, Germany)

\subsection{Assay method}

Twenty tablets were weighed and powdered. A quantity of powder containing $0.15 \mathrm{gm}$ of metformin- $\mathrm{HCl}$ were shaken for 15 minutes with $100 \mathrm{ml}$ of distilled water and filtered discarding the first $20 \mathrm{ml} .10 \mathrm{ml}$ of the resulting solution were diluted to $100 \mathrm{ml}$ and further $1 \mathrm{ml}$ and $5 \mathrm{ml}$ of the resulting solution were diluted to $100 \mathrm{ml}$ with distilled water. A standard solution of active metformin- $\mathrm{HCl}$ was also prepared to compare the drug content. Absorbance of the resulting solutions was measured at the maximum $233 \mathrm{~nm}$ and the content of metformin- $\mathrm{HCl}$ was calculated.

\subsection{Dissolution method}

A USP type-II six-vessel Dissolution Tester (Basket stirrer) with variable rotation speed was used for dissolution test. The basket rotation was set at $100 \mathrm{rpm}$ and the temperature was controlled at $37^{\circ} \mathrm{C} \pm 0.5^{\circ} \mathrm{C}$ using $900 \mathrm{ml}$ phosphate buffer of $\mathrm{pH} 6.8$ as chamber volume [18]. When the dissolution medium attained the bath temperature, one tablet was placed in each vessel. Six tablets were thus tested simultaneously from each of the products of metformin-HCl. Samples were collected after 30 minutes and filtered. $10 \mathrm{ml}$ of filtered aliquots were diluted to $100 \mathrm{ml}$ with distilled water and further $10 \mathrm{ml}$ of the resulting solutions were diluted to $100 \mathrm{ml}$ with distilled water and the absorbance were determined by UV spectrophotometer at $233 \mathrm{~nm}$ (Shimadzu, UV-1200, UV-VIS spectrophotometer). A standard solution of the active ingredient was also prepared to compare the drug release.

\subsection{Experimental animals}

A total number of 27 Wister albino rats (6-8 weeks age, 105-160 gm body weight) of either sex were purchased from animal house of International Centre for Diarrheal Disease Research, Bangladesh (ICDDR, B). Prior to the commencement of the experiment, all the rats were acclimatized to the new environmental condition for a period of one week. During the experimental period, rats were kept in a well-ventilated animal house at an ambient temperature $\left(25^{\circ} \mathrm{C}\right)$. They were supplied with standard pellets provided by 
ICDDR, B and fresh drinking water ad libitum. All animals were maintained with natural 12 hours light and dark cycle. Rats of similar gender were divided into 9 groups. Animal experiments were performed following the Helsinki declaration [19]

\subsection{Induction of diabetes}

Rats of group II-IX were rendered diabetic by intraperitoneal injection of freshly prepared solution of streptozotocin $(45 \mathrm{mg} / \mathrm{kg})$ in $0.1 \mathrm{~mol} / \mathrm{L}$ citrate buffer $(\mathrm{pH} 4.5)$ in a volume of 1 $\mathrm{ml} / \mathrm{kg}$ [20]. After 48 hours of treatment, blood glucose content was measured by using glucometer from the tail vein of the rats. Rats with blood glucose levels above 11.1 $\mathrm{mmol} / \mathrm{L}$ were considered as diabetic animal model for the study. Before induction of diabetes, baseline blood glucose level was also measured.

\subsection{Preparation of dose}

Ten tablets of each of the six products were weighed and powdered. Doses were prepared according to the body weight of the rats and were administered orally as $110 \mathrm{mg} / \mathrm{kg}$ body weight. Powder containing equivalent amount of drug for each rats were weighed accurately and dissolved in small amount of distilled water and then administered. Standard metformin- $\mathrm{HCl}$ dose was prepared by weighing required amount of metformin- $\mathrm{HCl}$ powder directly and dissolving in distilled water.

\subsection{Administration on diabetic rats}

Group I served as a non-diabetic control while group II to IX were rendered diabetic. Group II served as diabetic control. Standard metformin-HCl was administered orally to Group III rats. Product F1 was administered to group IV and F2, F3, F4, F5 and F6 were administered to group V, VI, VII, VIII and IX rats, respectively.

\subsection{Test of blood glucose level}

After 5 hours from the time of drug administration blood glucose levels were tested for every rats of each group. The tail vein of rats was pricked with a needle and withdrawing blood was tested with the help of glucometer.

\subsection{Statistical analysis}

Data were expressed as mean \pm standard error of mean (SEM). Statistical study was performed by Student's $t$-test and the values were considered statistically significant at $p<0.05$. 


\section{Results and Discussion}

\subsection{All tested products comply the content and dissolution specification of USP}

The average content of metformin and dissolution test results of all of the six products is given in Table 1. All samples contain drug within the USP specified limit (95-105\%). The final dissolution data showed that all products comply with the USP specification (90-110\%).

Table 1. Contents of metformin- $\mathrm{HCl}$ and dissolution results of commercial metformin- $\mathrm{HCl}$ tablets.

\begin{tabular}{ccc}
\hline Sample code & Content (\%) & $\begin{array}{c}\text { Release of drug } \\
\text { within } 30 \text { minutes }(\%)\end{array}$ \\
\hline F1 & 95.09 & 100.24 \\
F2 & 98.36 & 100.00 \\
F3 & 98.54 & 100.24 \\
F5 & 98.18 & 98.01 \\
F6 & 96.73 & 104.95 \\
\hline
\end{tabular}

\subsection{The tested products demonstrated significant antihyperglycemic potentialities}

The therapeutic efficacy of the products F1, F2, F3, F4, F5 and F6 on blood glucose levels were investigated in the control and streptozotocin induced diabetic rats using metformin- $\mathrm{HCl}$ as standard anti-diabetic agents. The mean blood glucose level of normal and diabetic control rats were shown in Fig. 1 and that of the drug treated animals (after oral administration of a single dose) after 5 hours were shown in Fig. 2. It was shown that all the products significantly reduced the blood glucose of streptozotocin-induced diabetic rats. A significant reduction $(p<0.05)$ in blood glucose of 58.1, 53.2, 50.8, 77.0, 72.9, 49.1 and $71.3 \%$ were observed after 5 hours with single dose of products F1, F2, F3, F4, F5, F6 and standard metformin- $\mathrm{HCl}$, respectively. All of the products reduced blood glucose level in diabetic rats, which are consistent with that of standard drug metformin- $\mathrm{HCl}$. 


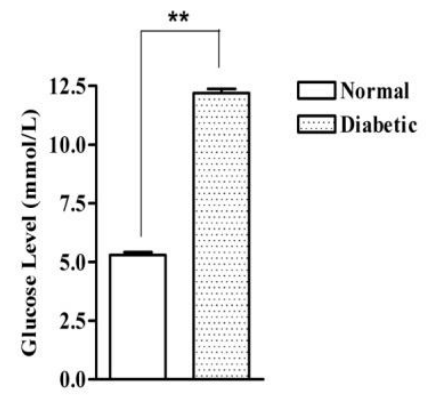

Fig. 1. Blood glucose levels of normal and diabetic rats. Each bar indicates the mean ( \pm SEM) blood glucose levels of three animals. "**” indicates $P<0.01$.

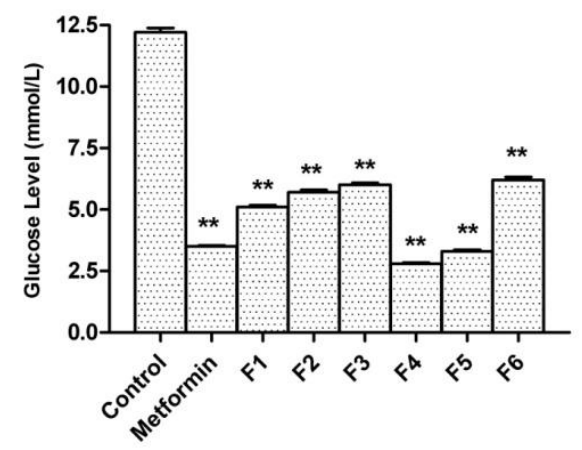

Fig. 2. Effects of products F1, F2, F3, F4, F5, F6 and standard metformin-HCl after oral administration in streptozotocin-induced diabetic rats. Each bar indicates the mean ( \pm SEM) blood glucose levels of three animals. There are significant differences from control in the mean glucose levels. “**” indicates $p<0.01$.

This study is a unique experimental scheme because it combined both pharmaceutical and pharmacological approach for bioequivalency assessment of metformin finished product. We have used animal model for successful biological evaluation of the metformin tablet, which was consistent with their active metformin content and dissolution properties and bioequivalence requirements [21]. Since diabetes is a disease that is not cured completely, it needs regular physical exercise, dieting, continuous monitoring and medication. Among oral anti-diabetic formulations, metformin- $\mathrm{HCl}$ is an ideal drug being used frequently. As reported recently around $10 \%$ of total population of Bangladesh is now suffering from diabetes [22]. According to WHO statistics 347 million people worldwide is suffering from diabetes, which is estimated to be the $7^{\text {th }}$ leading cause of death worldwide by 2030. As an ideal antidiabetic medicine, metformin tablet is supposed to be used by huge number of diabetic patients. From this point of view, it is quite easy to understand the importance of this study, which concerned with safety and efficacy for large number of population worldwide. 
Our study revealed that metformin- $\mathrm{HCl}$ tablets marketed by Bangladeshi companies fulfill their requirements to be an ideal product. They have shown potential blood glucose lowering efficacy in streptozotocin-induced hyperglycemic rats. Assay and dissolution data of all the products follow USP specification. So it is not excessive to comment that Bangladeshi pharma companies are producing quality metformin- $\mathrm{HCl}$ tablets, following the physical and chemical specifications such as mixing, temperature control, granulation time, addition of proper disintegrants, binders and other excipients. Our study has provided strong evidence that the tablet formulations of metformin- $\mathrm{HCl}$ marketed by Bangladeshi manufacturers conform all the standard specifications and can be used safely by the patients of diabetes in Bangladesh and abroad without any confusion in terms of efficacy unless intolerated.

\section{Acknowledgement}

We are grateful to Professor Dr. Shah Amran, Department of Pharmaceutical Chemistry, Dhaka University, for his useful comments on this manuscript. All authors read and approved the final manuscript. The authors have no conflict of interest. This work was partially supported by Ministry of National Science and Information and Communication Technology (NSICT), Bangladesh.

\section{References}

1. D. A. Greeene, A. A Sima, M. J. Stevens, E. L. Feldman, and S. A. Lattimer, Diab. Car. 15 (12),1902 (1992). http://dx.doi.org/10.2337/diacare.15.12.1902

2. L. Luzi, N. Engl. J. Med. 339, 115 (1998). http://dx.doi.org/10.1056/NEJM199807093390210

3. J. Larner, Insulin and Oral Hypoglycaemic Drugs, Glucagon. In: The Pharmacological Basis of Therapeutics, A. G. Gilman and T. W Goodman (ed.) (Murad, Macmillan, New York.1985).

4. J. K .Grover, S. Yadav, and V. Vats, J. Ethnopharmacol. 81, 81 (2002). http://dx.doi.org/10.1016/S0378-8741(02)00059-4

5. M. A. Islam, M. A. Akhtar, M. R. I. Khan, M. S. Hossain, M. K. Alam, M. I. I. Wahed, B. M. Rahman, A. S. M. Anisuzzaman, S. M. Shaheen, and M. Ahmed, J. Sci. Res. 1 (2), 334 (2009). : DOI: $10.3329 /$ jsr.v1i2.1075

6. M. R. I. Khan, M. A. Islam, M. S. Hossain, M. Asadujjaman, M. I. I. Wahed, B. M. Rahman, A. S. M Anisuzzaman, S. M. Shaheen and M. Ahmed, J. Sci. Res. 2 (1), 158 (2010). http://dx.doi.org/10.3329/jsr.v5i1.10252

7. R. Sharmin, M. R. I. Khan, Most. A. Akhtar, A. Alim, M. A. Islam, A. S. M. Anisuzzaman, M. Ahmed, J. Sci. Res. 5 (1), 161 (2013). http://dx.doi.org/10.3329/jsr.v5i1.10252

8. UK Prospective Diabetes Study (UKPDS) Group, Lancet 352, 854 (1998). http://dx.doi.org/10.1016/S0140-6736(98)07037-8

9. A. Karim, M. Slater, M. D. Bradford, M. L.Schawartz, M. Z. Zhao, C. Cao, and A. Laurent, J. Clin. Pharmacol. 47 (1), 37 (2007). http://dx.doi.org/10.1177/0091270006293755

10. R. A. Defronzo and A. M. Goodman, N. Engl. J. Med. 333, 341 (1995). http://dx.doi.org/10.1056/NEJM199508313330902

11. C. Bailey, Diabet. Car. 15, 755 (1992). http://dx.doi.org/10.2337/diacare.15.6.755

12. S. Bang, Y. Chen, R. S. Ahima, and S. F. Kim. Mol. Endocrin. 28 (7), 1186 (2014). http://press.endocrine.org/doi/pdf/10.1210/me.2014-1134

13. M. Stumvall, N. Nurjhan, G. Perriello, G. Daily, and J. Gerich, N. Engl. J. Med. 333, 550 (1995). http://dx.doi.org/10.1056/NEJM199508313330903 
14. M. A. Habib and M. Z. Alam, J. Bus. Tech. 6 (1), 61 (2011). http://dx.doi.org/10.3329/jbt.v6i1.9995

15. A.B.M. Faroque, Bangl. J. Bioethics. 2 (1), 18 (2011). http://dx.doi.org/10.3329/bioethics.v2i1.9813

16. J. W. Collier, R. B. Shah, A. Gupta, V. Sayeed, M. J. Habib, and M. A. Khan, AAPS Pharm. Sci. Tech. 11, 818 (2010). http://dx.doi.org/10.1208/s12249-010-9434-8

17. M. Z. Sultan, M. A. Mazid and M. A. Rashid, J. Sci. Res. 3(2), 383(2011). http://dx.doi.org/10.3329/jsr.v3i2.7024

18. United States Pharmacopoeia $\mathbf{2 4}^{\text {th }}$ Edition, The United States Pharmacopoeial Convention, Inc. Rockville, MD (2000).

19. WMA statement on animal use in Biomedical research, $57^{\text {th }}$ WMA General Assembly, Pilaneberg, South Africa, October, 2006.

http://www.wma.net/en/30publications/10policies/a18/index.html.pdf?print-media-type\&footerright=[page]/[toPage]

20. O. Siddique, Y. Sun, J. C. Lin, and Y. W. Chein, J Pharm Sci. 76, 341 (1987). http://dx.doi.org/10.1002/jps.2600760416

21. A. A, Talla, S. H. Akrawi, L. T. Tolley, S. H. Sioud, M. F. Zaater, and A. -H, M, Emwas, Drug. Des. Devel. Ther. 5, 427 (2011). http://dx.doi.org/10.2147/DDDT.S24504

22. B. Bhowmik, F. Afsana, L. M. Diep, S. B.Munir, E. Wright, S. Mahmood, A. K. A. Khan, and A. Hussain. Diabetes Metab. J. 37, 46 (2013).

http://www.ncbi.nlm.nih.gov/pmc/articles/PMC3638228/pdf/dmj-37-153.pdf 